\title{
Disturbance due to mechanical and thermal sources in a generalized thermo-microstretch elastic half space
}

\author{
RAJNEESH KUMAR and SUNITA DESWAL \\ Department of Mathematics, Kurukshetra University, Kurukshetra 136 119, India \\ e-mail: search@ vidya.kuk.ernet.in
}

MS received 26 June 2000; revised 6 June 2001

\begin{abstract}
Disturbances caused by impulsive concentrated mechanical and thermal sources in a homogeneous, isotropic generalized thermo-microstretch elastic medium are studied by the use of Laplace-Hankel transform techniques. The integral transforms are inverted using a numerical technique. Analytical expressions for displacement components, stress, couple stress, microstress and temperature field are derived for different models of generalized thermoelasticity and illustrated graphically. These results for stresses and displacements can be used in estimating the effects of a surface pressure wave. Stretch and micropolar effects on various expressions obtained analytically are also depicted graphically.
\end{abstract}

Keywords. Thermo-microstretch; mechanical/thermal/impulsive sources; Laplace-Hankel transforms.

\section{Introduction}

Modern engineering components are often made up of materials possessing internal structure. Polycrystalline materials and materials with fibrous or coarse grain structure belong to this category. Classical elasticity is not adequate to model the behaviour of such materials. "Micropolar elasticity", termed so by Eringen (1966a), is used to describe deformation of elastic media with oriented particles. A micropolar continuum is a collection of interconnected particles in the form of small rigid bodies undergoing both translational and rotational motions. Typical examples of such materials are granular media and multimolecular bodies, whose microstructures play an evident part in their macroscopic responses. The physical nature of these materials needs an asymmetric description of deformation. Micropolar theories have been developed by Eringen $(1966,1976)$ for elastic solids, fluids and also for non-local polar fields, and are now universally accepted.

To enhance the domain of application of micropolar elasticity, Eringen (1971, 1990b) included microstructural expansions and contractions as a linear theory. A microstretch continuum can model composite materials reinforced with chopped elastic fibres and various porous solids. The material points of these bodies can stretch and contract independently of

$\overline{\mathrm{A}}$ list of symbols is given at the end of the paper 
their translations and rotations. The theory of microstretch elastic bodies is a generalization of the micropolar theory.

The coupled theory of thermoelasticity has been extended by including the thermal relaxation time in the constitutive equations by Lord \& Shulman (1967) and Green \& Lindsay (1972). These theories eliminate the paradox of infinite velocity of heat propagation and are termed generalized theories of thermoelasticity. There exists the following differences between the two theories.

(a) The Lord-Shulman theory $(\mathrm{L}-\mathrm{S})$ involves one relaxation time of thermoelastic process $\left(t_{0}\right)$ and that of Green \& Lindsay (G-L) involves two relaxation times $\left(t_{0}, t_{1}\right)$.

(b) The L-S energy equation involves first and second time derivatives of strain, whereas the corresponding equation in G-L theory needs only the first time derivative of strain.

(c) In the linearized case, according to the approach of Green and Lindsay, heat cannot propagate with finite speed unless the stresses depend on the temperature velocity, whereas according to Lord and Shulman the heat can propagate with finite speed even though the stresses there are independent of the temperature velocity.

Experimental evidence available is in favour of finite heat propagation speed. Generalized thermoelasticity theories are therefore more realistic than the conventional Fourier theory in dealing with certain practical problems involving very large heat fluxes and/or short time intervals like those occurring in laser heating and energy channels. The linear theory of micropolar thermoelasticity was developed by extending the theory of micropolar continua to include thermal effects by Eringen (1970) and Nowacki (1966). The theory of thermo-microstretch elastic solids was developed by Eringen (1990). Recently many authors discussed different type of problems in generalized thermoelastic medium (Ciarletta 1999; Chandrasekharaiah \& Srinath 2000), microstretch elastic medium (Iesan \& Quintanilla 1994; Iesan \& Nappa 1995; Iesan \& Pompei 1995; Iesan 1997; De Cicco \& Nappa 1997, 1999), thermo-microstretch elastic solids (Bofill \& Quintanilla 1995; Ciarletta 1999) and in generalized thermo-microstretch elastic medium (Singh \& Kumar 1998). The objective of this paper is to study the disturbance due to mechanical and thermal sources acting at a point on the surface $z=0$ in the generalized thermo-microstretch elastic half space by applying integral transform techniques. Various components of stresses and displacements and the temperature field are calculated for an impulsive source on the surface.

\section{Formulation of the problem and the solution}

A homogeneous, isotropic, generalized thermo-microstretch elastic solid occupying the semiinfinite region $z>0$, is considered in an undisturbed state and initially at uniform temperature $T_{0}$. We take the origin of the cylindrical co-ordinate system $(r, \theta, z)$ on the surface $z=0$ and $z$-axis pointing vertically into the medium. Let $T(r, z, t)$ be the change in temperature of the medium. A mechanical source or thermal impulsive source is assumed to act at the origin of the cylindrical co-ordinate system having isothermal boundary. Following Eringen (1990), Lord \& Shulman (1967) and Green \& Lindsay (1972), the field equations and constitutive relations for a homogeneous, isotropic generalized thermo-microstretch elastic solid without body forces, body couples, heat sources and stretch force, can be written as

$$
(\lambda+\mu) \nabla(\nabla \cdot \mathbf{u})+(\mu+K) \nabla^{2} \mathbf{u}+K \nabla x \boldsymbol{\phi}-v\left(1+t_{1} \frac{\partial}{\partial t}\right) \nabla T+\lambda_{o} \nabla \phi^{*}=\rho \frac{\partial^{2} \mathbf{u}}{\partial t^{2}},
$$




$$
\begin{aligned}
& (\alpha+\beta+\gamma) \nabla(\nabla . \boldsymbol{\phi})-\gamma \nabla x(\nabla x \boldsymbol{\phi})+K \nabla x \mathbf{u}-2 K \boldsymbol{\phi}=\rho j \frac{\partial^{2} \boldsymbol{\phi}}{\partial t^{2}}, \\
& \rho C^{*}\left(\frac{\partial T}{\partial t}+t_{0} \frac{\partial^{2} T}{\partial t^{2}}\right)+v T_{0}\left(\frac{\partial}{\partial t}+\delta_{1 k} t_{0} \frac{\partial^{2}}{\partial t^{2}}\right) \nabla \cdot \mathbf{u}+v_{1} T_{0} \frac{\partial \phi^{*}}{\partial t}=K^{*} \nabla^{2} T, \\
& c_{6}^{2} \nabla^{2} \phi^{*}+\overline{v_{1}}\left(T+t_{1} \frac{\partial T}{\partial t}\right)-c_{7}^{2} \phi^{*}-c_{8}^{2} \nabla \cdot \mathbf{u}=\frac{\partial^{2} \phi^{*}}{\partial t^{2}},
\end{aligned}
$$

and

$$
\begin{aligned}
t_{i j} & =\lambda u_{r, r} \delta_{i j}+\mu\left(u_{i j}+u_{j, i}\right)+K\left(u_{j, i}-\epsilon_{i j r} \phi_{r}\right)-v\left(T+t_{1} \frac{\partial T}{\partial t}\right) \delta_{i j}+\lambda_{o} \delta_{i j} \phi^{*}, \\
m_{i j} & =\alpha \phi_{r, r} \delta_{i j}+\beta \phi_{i j}+\gamma \phi_{j, i}, \quad \lambda_{k}=\alpha_{o} \phi_{k}^{*},
\end{aligned}
$$

where

$$
\begin{aligned}
\nu & =(3 \lambda+2 \mu+K) \alpha_{t_{1}}, \quad \overline{\nu_{1}}=\frac{2 \nu_{1}}{9 \rho j}, \quad \nu_{1}=(3 \lambda+2 \mu+K) \alpha_{t_{2}}, \\
c_{6}^{2} & =\frac{2 \alpha_{0}}{3 \rho j}, \quad c_{7}^{2}=\frac{2 \lambda_{1}}{9 \rho j}, \quad c_{8}^{2}=\frac{2 \lambda_{0}}{9 \rho j},
\end{aligned}
$$

where the symbols have their usual meanings. The constants $v$ and $v_{1}$ depend on mechanical as well as thermal properties of the body. For the Lord-Shulman $(\mathrm{L}-\mathrm{S})$ theory, $t_{1}=0, k=1$ and for the Green-Lindsay $(\mathrm{G}-\mathrm{L})$ theory $t_{1}>0$ and $k=2$. The thermal relaxations $t_{0}$ and $t_{1}$ satisfy the inequality $t_{1} \geq t_{0} \geq 0$ in G-L theory.

The necessary and sufficient conditions for the internal energy to be non-negative, as given by Eringen (1990), are

$$
\begin{aligned}
& 3 \lambda+2 \mu+K \geq\left(3 \lambda_{0}^{2} / \lambda_{1}\right), \quad 2 \mu+K \geq 0, \quad K \geq 0, \\
& 3 \alpha+\beta+\gamma \geq 0, \quad \gamma+\beta \geq 0, \quad \gamma-\beta \geq 0, \quad \alpha_{0} \geq 0, \quad \lambda_{1} \geq 0 .
\end{aligned}
$$

For axisymmetric problems, we assume,

$$
\mathbf{u}=\left(u_{r}, 0, u_{z}\right), \quad \phi=\left(0, \phi_{\theta}, 0\right) .
$$

We introduce the dimensionless quantities,

$$
\begin{aligned}
& r^{\prime}=\frac{\omega^{*}}{c_{2}} r, z^{\prime}=\frac{\omega^{*}}{c_{2}} z, t^{\prime}=\omega^{*} t, t_{1}^{\prime}=\omega^{*} t_{1}, t_{0}^{\prime}=\omega^{*} t_{0}, u_{r}^{\prime}=\frac{\rho \omega^{*} c_{2}}{v T_{0}} u_{r}, \\
& u_{z}^{\prime}=\frac{\rho \omega^{*} c_{2}}{\nu T_{0}} u_{z}, \phi_{\theta}^{\prime}=\frac{\rho c_{2}^{2}}{v T_{0}} \phi_{\theta}, \phi^{*}=\frac{\rho c_{2}^{2}}{v T_{0}} \phi^{*}, t_{i j}^{\prime}=\frac{1}{v T_{0}} t_{i j}, m_{i j}^{\prime}=\frac{\omega^{*}}{c_{2} v T_{0}} m_{i j}, \\
& \lambda_{z}^{\prime}=\frac{\omega^{*}}{c_{2} v T_{0}} \lambda_{z}, T^{\prime}=\frac{T}{T_{0}}
\end{aligned}
$$

where

$$
\omega^{*}=\rho C^{*} c_{2}^{2} / K^{*}, \quad c_{2}^{2}=\mu / \rho,
$$


In (1)-(4), we get (after suppressing the primes)

$$
\begin{aligned}
& \frac{(\mu+K)}{\rho c_{2}^{2}}\left(\nabla^{2}-\frac{1}{r^{2}}\right) u_{r}+\frac{(\lambda+\mu)}{\rho c_{2}^{2}} \frac{\partial e}{\partial r}-\frac{K}{\rho c_{2}^{2}} \frac{\partial \phi_{\theta}}{\partial z}-\left(1+t_{1} \frac{\partial}{\partial t}\right) \frac{\partial T}{\partial r}+\frac{\lambda_{0}}{\rho c_{2}^{2}} \frac{\partial \phi^{*}}{\partial r}=\frac{\partial^{2} u_{r}}{\partial t^{2}} \\
& \frac{(\mu+K)}{\rho c_{2}^{2}} \nabla^{2} u_{z}+\frac{(\lambda+\mu)}{\rho c_{2}^{2}} \frac{\partial e}{\partial z}+\frac{K}{\rho c_{2}^{2} r} \frac{\partial}{\partial r}\left(r \phi_{\theta}\right)-\left(1+t_{1} \frac{\partial}{\partial t}\right) \frac{\partial T}{\partial r}+\frac{\lambda_{0}}{\rho c_{2}^{2}} \frac{\partial \phi^{*}}{\partial z}=\frac{\partial^{2} u_{z}}{\partial t^{2}} \\
& \left(\nabla^{2}-\frac{1}{r^{2}}\right) \phi_{\theta}-\frac{2 K c_{2}^{2}}{\gamma \omega^{* 2}} \phi_{\theta}+\left(\frac{K c_{2}^{2}}{\gamma \omega^{* 2}}\right)\left(\frac{\partial u_{r}}{\partial z}-\frac{\partial u_{z}}{\partial r}\right)=\frac{\rho j c_{2}^{2}}{\gamma} \frac{\partial^{2} \phi_{\theta}}{\partial t^{2}} \\
& \nabla^{2} T-\left(\frac{\partial T}{\partial t}+t_{0} \frac{\partial^{2} T}{\partial t^{2}}\right)-\left(\frac{v^{2} T_{0}}{\rho K^{*} \omega^{*}}\right)\left(\frac{\partial}{\partial t}+\delta_{l k} t_{0} \frac{\partial^{2}}{\partial t^{2}}\right) e=\left(\frac{v v_{l} T_{0}}{\rho K^{*} \omega^{*}}\right) \frac{\partial \phi^{*}}{\partial t} \\
& \left(\frac{c_{6}^{2}}{c_{2}^{2}} \nabla^{2}-\frac{c_{7}^{2}}{\omega^{* 2}}-\frac{\partial^{2}}{\partial t^{2}}\right) \phi^{*}-\left(\frac{c_{8}^{2}}{\omega^{* 2}}\right) e+\frac{2 v_{1} c_{2}^{2}}{9 v \omega^{* 2} j}\left(1+t_{1} \frac{\partial}{\partial t}\right) T=0
\end{aligned}
$$

where

$$
\nabla^{2}=\frac{\partial^{2}}{\partial r^{2}}+\frac{1}{r} \frac{\partial}{\partial r}+\frac{\partial^{2}}{\partial z^{2}}, \quad e=\frac{1}{r} \frac{\partial}{\partial r}\left(r u_{r}\right)+\frac{\partial u_{z}}{\partial z}
$$

Assuming the potentials $\Phi, \Psi$ and $\Gamma$ defined by the relations in non-dimensional form,

$$
u_{r}=\frac{\partial \Phi}{\partial r}+\frac{\partial^{2} \Psi}{\partial r \partial z}, \quad u_{z}=\frac{\partial \Phi}{\partial z}-\left(\nabla^{2}-\frac{\partial^{2}}{\partial z^{2}}\right) \Psi, \quad \phi_{\theta}=-\frac{\partial \Gamma}{\partial r}
$$

and inserting (16) in (11)-(15), we obtain

$$
\begin{aligned}
& \left(\nabla^{2}-a_{0} \frac{\partial^{2}}{\partial t^{2}}\right) \Phi-a_{0}\left(1+t_{1} \frac{\partial}{\partial t}\right) T+a_{5} \phi^{*}=0, \\
& \left(\nabla^{2}-a_{3} \frac{\partial^{2}}{\partial t^{2}}\right) \Psi+a_{4} \Gamma=0, \\
& \left(\nabla^{2}-2 a_{1}-a_{2} \frac{\partial^{2}}{\partial t^{2}}\right) \Gamma-a_{1} \nabla^{2} \Psi=0, \\
& \left(\nabla^{2}-\frac{\partial}{\partial t}-t_{0} \frac{\partial^{2}}{\partial t^{2}}\right) T-\epsilon\left(\frac{\partial}{\partial t}+\delta_{1 k} t_{0} \frac{\partial^{2}}{\partial t^{2}}\right) \nabla^{2} \Phi-\epsilon_{1} \frac{\partial \phi^{*}}{\partial t}=0, \\
& a_{9}\left(1+t \frac{\partial}{\partial t}\right) T-a_{8} \nabla^{2} \Phi+\left(a_{6} \nabla^{2}-a_{7}-\frac{\partial^{2}}{\partial t^{2}}\right) \phi^{*}=0 .
\end{aligned}
$$


where

$$
\begin{aligned}
& a_{1}=\frac{K c_{2}^{2}}{\gamma \omega^{* 2}}, a_{2}=\frac{\rho j c_{2}^{2}}{\gamma}, a_{3}=\frac{\rho c_{2}^{2}}{\mu+K}, a_{4}=\frac{K}{\mu+K}, a_{5}=\frac{\lambda_{o}}{\lambda+2 \mu+K}, \\
& a_{6}=\frac{c_{6}^{2}}{c_{2}^{2}}, a_{7}=\frac{c_{7}^{2}}{\omega^{* 2}}, a_{8}=\frac{c_{8}^{2}}{\omega^{* 2}}, a_{9}=\frac{2 v_{1} c_{2}^{2}}{9 \nu \omega^{* 2} j}, a_{0}=\frac{c_{2}^{2}}{c_{1}^{2}}, \\
& c_{1}^{2}=\frac{\lambda+2 \mu+K}{\rho}, \in=\frac{\nu^{2} T_{0}}{\rho K^{*} \omega^{*}}, \in_{1}=\frac{\nu v_{1} T_{0}}{\rho K^{*} \omega^{*}} .
\end{aligned}
$$

Applying Laplace and Hankel transforms defined by

$$
\begin{aligned}
& \bar{f}(p)=\int_{0}^{\infty} e^{-p t} f(t) \mathrm{d} t \quad \text { and } \\
& \hat{f}(q, z, p)=\int_{0}^{\infty} \bar{f}(r, z, p) r J_{n}(r q) \mathrm{d} r,
\end{aligned}
$$

where $J_{n}()$ is the Bessel function of the first kind of order $n$, on (17)-(21) and then eliminating $\hat{T}, \hat{\phi}^{*}$ and $\hat{\Gamma}$ from the resulting expressions, we obtain

$$
\begin{aligned}
& {\left[\nabla^{\prime 6}+A \nabla^{\prime 4}+B \nabla^{\prime 2}+C\right][\hat{\Phi}]=0 \text { and }} \\
& {\left[\frac{d^{4}}{d z^{4}}+E \frac{d^{2}}{d z^{2}}+F\right][\hat{\Psi}]=0,}
\end{aligned}
$$

where

$$
\begin{aligned}
A & =-\left(a_{0} p^{2}+d_{3}+d_{7}\right)+a_{5} d_{8}-\in d_{1} d_{2}, \\
B & \left.=d_{3} d_{7}+p^{2} a_{0}\left(d_{3}+d_{7}\right)-\in a_{5} d_{2} d_{5} d_{9}+\epsilon_{1} \quad p\left(d_{5} d_{9}-d_{1} d_{8}\right)-a_{5} d_{3} d_{8}\right)+\in d_{1} d_{2} d_{7}, \\
C & =-p^{2} a_{0}\left(d_{3} d_{7}+\epsilon_{1} p d_{5} d_{9}\right), \quad E=-2 q^{2}-2 a_{1}-p^{2}\left(a_{2}+a_{3}\right)+a_{1} a_{4} \\
F & =q^{4}+q^{2}\left[2 a_{1}+p^{2}\left(a_{2}+a_{3}\right)-a_{1} a_{4}\right]+a_{3} p^{2}\left(2 a_{1}+a_{2} p^{2}\right) \\
d_{1} & =a_{0}\left(1+p t_{1}\right), \quad d_{2}=p+\delta_{1 k} t_{0} p^{2}, \quad d_{3}=p+t_{0} p^{2}, \quad d_{5}=1+p t_{1} \\
d_{7} & =\frac{a_{7}+p^{2}}{a_{6}}, \quad d_{8}=\frac{a_{8}}{a_{6}}, \quad d_{9}=\frac{a_{9}}{a_{6}}, \quad \nabla^{\prime 2}=\frac{\mathrm{d}^{2}}{\mathrm{~d} z^{2}}-q^{2}
\end{aligned}
$$

Equation (24) can be written in the form

$$
\left[\nabla^{* 3}+A \nabla^{* 2}+B \nabla^{*}+C\right][\hat{\Phi}]=0, \text { where } \nabla^{*}=\nabla^{\prime 2} .
$$

Using Cardon's method to solve (27), we obtain

$$
s^{3}+3 H s+G=0,
$$


where

$$
s=\nabla^{*}+\frac{A}{3}, \quad H=\frac{B}{3}-\frac{A^{2}}{9}, \quad G=\frac{2 A^{3}}{27}-\frac{A B}{3}+C .
$$

Since $\Delta\left(=G^{2}+4 H^{3}\right)$ is negative, we obtain the three real roots of (28), as

$$
s_{n}=2(-H)^{1 / 2} \cos \left(\frac{\phi+2 \pi(n-1)}{3}\right), \quad(n=1,2,3),
$$

where

$$
\left.\phi=\tan ^{-1}(|\Delta|)^{1 / 2} /-G\right) .
$$

Hence

$$
\nabla_{n}^{*}=s_{n}-(A / 3) .
$$

Equation (32) can be written as

$$
\left(\frac{d^{2}}{d z^{2}}-\xi_{n}^{2}\right) \hat{\Phi}=0
$$

where

$$
\xi_{n}^{2}=q^{2}+s_{n}-\frac{A}{3}, \quad(n=1,2,3),
$$

and $\xi_{1}, \xi_{2}, \xi_{3}$ correspond to the longitudinal displacement wave, thermal wave and longitudinal microstretch wave respectively.

The roots of (25) are given as

$$
\xi_{4,5}^{2}=\left[-E \pm \sqrt{E^{2}-4 F}\right] / 2 .
$$

The solutions of (24) and (25), satisfying the radiation conditions at infinity, are

$$
\begin{aligned}
\hat{\Phi} & =A_{1} \exp \left(-\xi_{1} z\right)+A_{2} \exp \left(-\xi_{2} z\right)+A_{3} \exp \left(-\xi_{3} z\right), \\
\hat{T} & =b_{1}^{*} A_{1} \exp \left(-\xi_{1} z\right)+b_{2}^{*} A_{2} \exp \left(-\xi_{2} z\right)+b_{3}^{*} A_{3} \exp \left(-\xi_{3} z\right), \\
\hat{\phi}^{*} & =a_{1}^{*} A_{1} \exp \left(-\xi_{1} z\right)+a_{2}^{*} A_{2} \exp \left(-\xi_{2} z\right)+a_{3}^{*} A_{3} \exp \left(-\xi_{3} z\right), \\
\hat{\Psi} & =A_{4} \exp \left(-\xi_{4} z\right)+a_{5} \exp \left(-\xi_{5} z\right), \\
\hat{\Gamma} & =a_{4}^{*} A_{4} \exp \left(-\xi_{4} z\right)+a_{5}^{*} A_{5} \exp \left(-\xi_{5} z\right),
\end{aligned}
$$

where

$$
\begin{array}{r}
a_{1}^{*}=\left(-\xi_{i}^{2}-q^{2}-p^{2} a_{0}\right) /\left(d_{1} N_{i}-a_{5}\right), \quad a_{4,5}^{*}=-\frac{1}{a_{4}}\left[\xi_{4,5}^{2}-q^{2}-a_{3} p^{2}\right], \\
b_{i}^{*}=\frac{1}{d_{1}}\left[a_{5} a_{i}^{*}+\xi_{i}^{2}-q^{2}-p^{2} a_{0}\right], \quad N_{i}=\left[\frac{d_{8} \in_{1} p+\epsilon d_{2}\left(\xi_{i}^{2}-q^{2}-d_{7}\right)}{d_{8}\left(\xi_{i}^{2}-q^{2}-d_{3}\right)-\epsilon d_{2} d_{5} d_{9}}\right], \\
(i=1,2,3) .
\end{array}
$$




\section{Boundary conditions}

\subsection{Case I. Instantaneous mechanical source acting on the surface}

The plane boundary is subjected to an instantaneous normal point force and the boundary surface is isothermal. Therefore, the boundary conditions in this case are

$$
t_{z z}=[-P \delta(r) \delta(t)] / 2 \pi r, t_{z r}=m_{z \theta}=T=\lambda_{z}=0 \text { at } z=0,
$$

where $\delta()$ denotes the Dirac delta function and $P$ is the magnitude of the force. Making use of (5), (6), (10) and (16) in the boundary conditions (42), applying the transforms defined by (23) and then substituting (36)-(40) in the resulting expressions, we obtain the expressions for displacement components, stresses, temperature field and microstress as

$$
\begin{aligned}
\hat{u}_{r} & =\left(1 / \Delta_{0}\right)\left[-q\left(\Delta_{1} e^{-\xi_{1} z}+\Delta_{2} e^{-\xi_{2} z}+\Delta_{3} e^{-\xi_{3} z}\right)+q\left(\xi_{4} \Delta_{4} e^{-\xi_{5} z}+\xi_{5} \Delta_{5} e^{-\xi_{5} z}\right)\right] \\
\hat{u}_{z} & =\left(1 / \Delta_{0}\right)\left[-\xi_{1} \Delta_{1} e^{-\xi_{1} z}-\xi_{2} \Delta_{2} e^{-\xi_{2} z}-\xi_{3} \Delta_{3} e^{-\xi_{3} z}+q_{2}\left(\Delta_{4} e^{-\xi_{4} z}+\Delta_{5} e^{-\xi_{5} z}\right)\right] \\
\hat{t}_{z z} & =\left(1 / \Delta_{0}\right)\left[f_{1} \Delta_{1} e^{-\xi_{1} z}+f_{2} \Delta_{2} e^{-\xi_{2} z}+f_{3} \Delta_{3} e^{-\xi_{3} z}-b_{2} q^{2}\left(\xi_{4} \Delta_{4} e^{-\xi_{4} z}+\xi_{5} \Delta_{5} e^{-\xi_{5} z}\right)\right], \\
\hat{t}_{z r} & =\left(q / \Delta_{0}\right)\left[b_{2} \xi_{1} \Delta_{1} e^{-\xi_{1} z}+b_{2} \xi_{2} \Delta_{2} e^{-\xi_{2} z}+b_{2} \xi_{3} \Delta_{3} e^{-\xi_{3} z}+f_{4} \Delta_{4} e^{-\xi_{4} z}+f_{5} \Delta_{5} e^{-\xi_{5} z}\right], \\
\hat{m}_{z \theta} & =\left(-b_{6} q / \Delta_{0}\right)\left[\xi_{4} a_{4}^{*} \Delta_{4} e^{-\xi_{4} z}+\xi_{5} a_{5}^{*} \Delta_{5} e^{-\xi_{5} z}\right], \\
\hat{T} & =\left(1 / \Delta_{0}\right)\left[b_{1}^{*} \Delta_{1} e^{-\xi_{1} z}+b_{2}^{*} \Delta_{2} e^{-\xi_{2} z}+b_{3}^{*} \Delta_{3} e^{-\xi_{3} z}\right], \\
\hat{\lambda}_{z} & =\left(-b_{7} / \Delta_{0}\right)\left[\xi_{1} a_{1}^{*} e^{-\xi_{1} z}+\xi_{2} a_{2}^{*} e^{-\xi_{2} z}+\xi_{3} a_{3}^{*} \Delta_{3} e^{-\xi_{3} z}\right],
\end{aligned}
$$

where

$$
\begin{aligned}
& \Delta_{0}= b_{1}^{*}\left[\xi_{2} a_{2}^{*}\left(\xi_{5} a_{5}^{*} e_{2}-\xi_{4} a_{4}^{*} e_{1}\right)+\xi_{3} a_{3}^{*}\left(\xi_{4} a_{4}^{*} e_{3}-\xi_{5} a_{5}^{*} e_{4}\right)\right]+ \\
& b_{2}^{*}\left[\xi_{1} a_{1}^{*}\left(\xi_{4} a_{4}^{*} e_{1}-\xi_{5} a_{5}^{*} e_{2}\right)+\xi_{3} a_{3}^{*}\left(\xi_{5} a_{5}^{*} e_{6}-\xi_{4} a_{4}^{*} e_{5}\right)\right]+ \\
& b_{3}^{*}\left[\xi_{1} a_{1}^{*}\left(\xi_{5} a_{5}^{*} e_{4}-\xi_{4} a_{4}^{*} e_{3}\right)+\xi_{2} a_{2}^{*}\left(\xi_{4} a_{4}^{*} e_{5}-\xi_{5} a_{5}^{*} e_{6}\right)\right], \\
& \Delta_{1}= P_{0} e_{7}\left(\xi_{3} b_{2}^{*} a_{3}^{*}-\xi_{2} b_{3}^{*} a_{2}^{*}\right), \quad \Delta_{2}=P_{0} e_{7}\left(\xi_{1} a_{1}^{*} b_{3}^{*}-\xi_{3} b_{1}^{*} a_{3}^{*}\right), \\
& \Delta_{3}= P_{0} e_{7}\left(\xi_{2} b_{1}^{*} a_{2}^{*}-\xi_{1} b_{2}^{*} a_{1}^{*}\right), \quad \Delta_{4}=P_{0} \xi_{5} a_{5}^{*} b_{2} \Delta_{6}, \quad \Delta_{5}=-P_{0} \xi_{4} a_{4}^{*} b_{2} \Delta_{6}, \\
& \Delta_{6}=- b_{1}^{*} \xi_{2} \xi_{3}\left(a_{3}^{*}-a_{2}^{*}\right)+b_{2}^{*} \xi_{1} \xi_{3}\left(a_{3}^{*}-a_{1}^{*}\right)-b_{3}^{*} \xi_{1} \xi_{2}\left(a_{2}^{*}-a_{1}^{*}\right), \\
& f_{i}=\left(b_{1}+b_{2}\right) \xi_{i}^{2}-b_{1} q^{2}-b_{i}^{*} d_{1}+b_{0} a_{i}^{*}, \quad i=1,2,3, \\
& f_{j}=-b_{2} \xi_{j}^{2}+b_{4}\left(\xi_{j}^{2}-q^{2}\right)-b_{5} a_{j}^{*}, \quad j=4,5 ; \quad P_{0}=P / 2 \pi, \\
& e_{1}=f_{3} f_{5}+q^{2} b_{2}^{2} \xi_{3} \xi_{5}, \quad e_{2}=f_{3} f_{4}+q^{2} b_{2}^{2} \xi_{3} \xi_{4}, \quad e_{3}=f_{2} f_{5}+q^{2} b_{2}^{2} \xi_{2} \xi_{5}, \\
& e_{4}=f_{2} f_{4}+q^{2} b_{2}^{2} \xi_{2} \xi_{4}, \quad e_{5}=f_{1} f_{5}+q^{2} b_{2}^{2} \xi_{1} \xi_{5}, \quad e_{6}=f_{1} f_{4}+q^{2} b_{2}^{2} \xi_{1} \xi_{4},
\end{aligned}
$$




$$
\begin{aligned}
& e_{7}=f_{5} \xi_{4} a_{4}^{*}-f_{4} \xi_{5} a_{5}^{*}, \quad b_{0}=\lambda_{0} / \rho c_{2}^{2}, \quad b_{1}=\lambda / \rho c_{2}^{2}, b_{2}=(2 \mu+K) / \rho c_{2}^{2}, \\
& b_{4}=\mu / \rho c_{2}^{2}, \quad b_{5}=K / \rho c_{2}^{2}, \quad b_{3}=b_{4}+b_{5}, \quad b_{6}=\left(\gamma / \rho c_{2}^{2}\right)\left(\omega^{*} / c_{2}\right)^{2}, \\
& b_{7}=\left(\alpha_{0} / \rho c_{2}^{2}\right)\left(\omega^{*} / c_{2}\right)^{2} .
\end{aligned}
$$

Subcases: (1) For L-S theory; $d_{1}$ and $d_{2}$ in (43)-(49), take the form

$$
d_{1}=a_{0}, \quad d_{2}=p+t_{0} p^{2}, \quad d_{5}=1 .
$$

(2) For G-Ls theory, $d_{1}$ and $d_{5}$ in (43)-(49) remain the same as given by (26) and $d_{2}$ takes the form

$$
d_{2}=p .
$$

Particular case 1: By taking $\alpha_{0}=\lambda_{0}=\lambda_{1}=0$ in (43)-(49), we obtain the expressions for displacement components, stresses and temperature field in a micropolar thermoelastic medium as

$$
\begin{aligned}
\hat{u}_{r} & =\left(1 / \Delta_{0}^{\prime}\right)\left[-q\left(\Delta_{1}^{\prime} e^{-\xi_{1}^{\prime} z}+\Delta_{2}^{\prime} e^{-\xi_{2}^{\prime} z}\right)+q\left(\xi_{4} \Delta_{4}^{\prime} e^{-\xi_{4}^{\prime} z}+\xi_{5} \Delta_{5}^{\prime} e^{-\xi_{5} z}\right)\right], \\
\hat{\mu}_{z} & =\left(1 / \Delta_{0}^{\prime}\right)\left[-\xi_{1}^{\prime} \Delta_{1}^{\prime} e^{-\xi_{1}^{\prime} z}-\xi_{2}^{\prime} \Delta_{2}^{\prime} e^{-\xi_{2}^{\prime} z}+q^{2}\left(\Delta_{4}^{\prime} e^{-\xi_{4} z}+\Delta_{5}^{\prime} e^{-\xi_{5} z}\right)\right], \\
\hat{t}_{z z} & =\left(1 / \Delta_{0}^{\prime}\right)\left[f_{1}^{\prime} \Delta_{1}^{\prime} e^{-\xi_{1}^{\prime} z}+f_{2} \Delta_{2}^{\prime} e^{-\xi_{2}^{\prime} z}-b_{2} q^{2}\left(\xi_{4} \Delta_{4}^{\prime} e^{-\xi_{4} z}+\xi_{5} \Delta_{5}^{\prime} e^{-\xi_{5} z}\right)\right], \\
\hat{t}_{z r} & =\left(q / \Delta_{0}^{\prime}\right)\left[b_{2}\left(\xi_{1}^{\prime} \Delta_{1}^{\prime} e^{-\xi_{1}^{\prime} z}+\xi_{2}^{\prime} \Delta_{2}^{\prime} e^{-\xi_{2}^{\prime} z}\right)+f_{4} \Delta_{4}^{\prime} e^{-\xi_{4} z}+f_{5} \Delta_{5}^{\prime} e^{-\xi_{5} z}\right], \\
\hat{m}_{z \theta} & =\left(-b_{6} q / \Delta_{0}^{\prime}\right)\left[\xi_{4} a_{4}^{*} \Delta_{4}^{\prime} e^{-\xi_{4} z}+\xi_{5} a_{5}^{*} \Delta_{5}^{\prime} e^{-\xi_{5} z}\right], \\
\hat{T} & =\left(1 / \Delta_{0}^{\prime}\right)\left[q_{1} \Delta_{1}^{\prime} e^{-\xi_{1}^{\prime} z}+q_{2} \Delta_{2}^{\prime} e^{-\xi_{2}^{\prime} z}\right],
\end{aligned}
$$

where

$$
\begin{array}{rlrl}
\Delta_{0}^{\prime} & =\xi_{4} a_{4}^{*}\left(q_{1} S_{1}-q_{2} S_{3}\right) \xi_{5} a_{5}^{*}\left(q_{1} S_{2}-q_{2} S_{4}\right), \\
\Delta_{1}^{\prime} & =-P_{0} q_{2}\left(f_{4} \xi_{5} a_{5}^{*}-f_{5} \xi_{4} a_{4}^{*}\right), & \Delta_{2}^{\prime}=P_{0} q_{1}\left(f_{4} \xi_{5} a_{5}^{*}-f_{5} \xi_{4} a_{4}^{*}\right), \\
\Delta_{4}^{\prime} & =P_{0} \xi_{5} a_{5}^{*} b_{2}\left(\xi_{1}^{\prime} q_{2}-\xi_{2}^{\prime} q_{1}\right), & \Delta_{5}^{\prime} & =P_{0} \xi_{4} a_{4}^{*} b_{2}\left(\xi_{2}^{\prime} q_{1}-\xi_{1}^{\prime} q_{2}\right), \\
S_{1} & =f_{2}^{\prime} f_{5}+b_{2}^{2} q^{2} \xi_{2}^{\prime} \xi_{5}, & S_{2}=f_{2}^{\prime} f_{4}+b_{2}^{2} q^{2} \xi_{2}^{\prime} \xi_{4}, \\
S_{3} & =f_{1}^{\prime} f_{5}+b_{2}^{2} q^{2} \xi_{1}^{\prime} \xi_{5}, & S_{4}=f_{1}^{\prime} f_{4}+b_{2}^{2} q^{2} \xi_{1}^{\prime} \xi_{4}, \\
\xi_{1,2}^{\prime 2} & =\left[-A^{\prime} \pm\left(A^{\prime 2}-4 B^{\prime}\right)^{1 / 2}\right] / 2, & A^{\prime}=-\left[2 q^{2}+d_{3}+a_{0} p^{2}+\in d_{1} d_{2}\right], \\
B^{\prime} & =q^{4}+q^{2}\left[d_{3}+p^{2} a_{0}+\in d_{1} d_{2}\right]+a_{0} d_{3} p^{2}, \quad q_{1,2}=\left(1 / d_{1}\right)\left[\xi_{1,2}^{\prime 2}-q^{2}-p^{2} a_{0}\right], \\
f_{i}^{\prime} & =\left(b_{1}+b_{2}\right) \xi_{i}^{\prime 2}-b_{1} q^{2}-q_{i} d_{1} / a_{0} ; \quad i=1,2 .
\end{array}
$$

(1) For $\mathrm{L}-\mathrm{S}$ theory, $d_{i}(i=1,2,5)$ in (53)-(58) are as defined by (51).

(2) For G-L theory, $d_{i}(i=1,2,5)$ in (53)-(58) are as defined by (52). 
Particular case 2: If we neglect the microstretch effect in (43)-(49) by putting $\alpha_{0}=\lambda_{0}=$ $\lambda_{1}=K=\beta=\gamma=0$, we obtain the expressions for displacement components, stresses and temperature field in a generalized thermoelastic medium as

$$
\begin{aligned}
& \hat{u}_{r}=\left(1 / \Delta_{0}^{*}\right)\left[-q\left(\Delta_{6} e^{-\xi_{1}^{\prime} z}+\Delta_{7} e^{-\xi_{2}^{\prime} z}\right)+q \xi_{3}^{\prime} \Delta_{8} e^{-\xi_{3}^{\prime} z}\right], \\
& \hat{u}_{z}=\left(1 / \Delta_{0}^{*}\right)\left[-\xi_{1}^{\prime} \Delta_{6} e^{-\xi_{1}^{\prime} z}-\xi_{2}^{\prime} \Delta_{7} e^{-\xi_{2}^{\prime} z}+q^{2} \Delta_{8} e^{-\xi_{3}^{\prime} z}\right], \\
& \hat{t}_{z z}=\left(1 / \Delta_{0}^{*}\right)\left[H_{1} \Delta_{6} e^{-\xi_{1}^{\prime} z}+H_{2} \Delta_{7} e^{-\xi_{2}^{\prime} z}+H_{3} \Delta_{8} e^{-\xi_{3}^{\prime} z}\right], \\
& \hat{t}_{z r}=\left(1 / \Delta_{0}^{*}\right)\left[G_{1} \Delta_{6} e^{-\xi_{1}^{\prime} z}+G_{2} \Delta_{7} e^{-\xi_{2}^{\prime} z}+G_{3} \Delta_{8} e^{-\xi_{3}^{\prime} z}\right], \\
& \hat{T}=\left(1 / \Delta_{0}^{*}\right)\left[q_{1} \Delta_{6} e^{-\xi_{1}^{\prime} z}+q_{2} \Delta_{7} e^{-\xi_{2}^{\prime} z}\right],
\end{aligned}
$$

where

$$
\begin{aligned}
& \Delta_{0}^{*}=q_{1}\left(H_{2} G_{3}-H_{3} G_{2}\right)-q_{2}\left(H_{1} G_{3}-H_{3} G_{1}\right), \\
& \Delta_{6}=P_{0} G_{3} q_{2}, \quad \Delta_{7}=-P_{0} G_{3} q_{1}, \quad \Delta_{8}=P_{0}\left(G_{2} q_{1}-G_{1} q_{2}\right), \\
& H_{i}=\left(b_{1}+b_{2}^{\prime}\right) \xi_{i}^{\prime 2}-b_{1} q^{2}-q_{i} d_{5}, \quad i=1,2, \\
& H_{3}=-b_{2}^{\prime} q^{2} \xi_{3}^{\prime}, \quad G_{i}=q b_{2}^{\prime} \xi_{i}^{\prime}, \quad i=1,2, \\
& G_{3}=-q b_{4}\left(\xi_{3}^{\prime 2}+q^{2}\right), \quad b_{2}^{\prime}=2 \mu / \rho c_{2}^{2}, \\
& \xi_{3}^{\prime 2}=q^{2}+a_{3}^{\prime} p^{2}, \quad a_{3}^{\prime}=\rho c_{2}^{2} / \mu .
\end{aligned}
$$

For $\mathrm{L}-\mathrm{S}$ and $\mathrm{G}-\mathrm{L}$ theories, $d_{i}(i=1,2,5)$ are given by (51) and (52) respectively.

\subsection{Case 2. Instantaneous thermal source acting on the surface}

When the plane boundary is stress-free and subjected to an instantaneous thermal point source, the boundary conditions are,

$$
t_{z z}=t_{z r}=m_{z \theta}=\lambda_{z}=0, T=P \delta(r) \delta(t) / 2 \pi r, \text { at } z=0,
$$

where $P$ is the constant temperature applied on the boundary and the boundary is kept stressfree. With the help of these boundary conditions (66) and following the procedure adopted in case 1 , the expressions for displacement components, force stresses, couple stress, microstress and temperature field are again given by (43)-(49) with $\Delta_{i}(i=1,2, \ldots, 6)$ replaced by $\Delta_{i}^{*}$, where

$$
\begin{aligned}
& \Delta_{1}^{*}=-P_{0}\left[\xi_{2} a_{2}^{*}\left(\xi_{4} a_{4}^{*} e_{1}-\xi_{5} a_{5}^{*} e_{2}\right)+\xi_{3} a_{3}^{*}\left(\xi_{5} a_{5}^{*} e_{4}-\xi_{4} a_{4}^{*} e_{3}\right)\right], \\
& \Delta_{2}^{*}=P_{0}\left[\xi_{1} a_{1}^{*}\left(\xi_{4} a_{4}^{*} e_{1}-\xi_{5} a_{5}^{*} e_{2}\right)+\xi_{3} a_{3}^{*}\left(\xi_{5} a_{5}^{*} e_{6}-\xi_{4} a_{4}^{*} e_{5}\right)\right], \\
& \Delta_{3}^{*}=-P_{0}\left[\xi_{1} a_{1}^{*}\left(\xi_{4} a_{4}^{*} e_{3}-\xi_{5} a_{5}^{*} e_{4}\right)+\xi_{2} a_{2}^{*}\left(\xi_{5} a_{5}^{*} e_{6}-\xi_{4} a_{4}^{*} e_{5}\right)\right], \\
& \Delta_{4}^{*}=-P_{0} \xi_{5} a_{5}^{*} b_{2} \Delta_{6}^{*}, \quad \Delta_{5}^{*}=P_{0} \xi_{4} a_{4}^{*} b_{2} \Delta_{6}^{*}, \\
& \Delta_{6}^{*}=\xi_{1} a_{1}^{*}\left(f_{2} \xi_{3}-f_{3} \xi_{2}\right)-\xi_{2} a_{2}^{*}\left(f_{1} \xi_{3}-f_{3} \xi_{1}\right)+\xi_{3} a_{3}^{*}\left(f_{1} \xi_{2}-f_{2} \xi_{1}\right) .
\end{aligned}
$$


Subcases: Replacing $\Delta_{i}(i=1, \ldots, 6)$ by $\Delta_{i}^{*}$ defined by (67), in (43)-(49), we obtain the expressions for displacement components, stresses, temperature distribution and microstress.

(1) For L-S theory with $d_{1}, d_{2}$ and $d_{5}$ defined by (51)

(2) For G-L theory with $d_{1}, d_{2}$ and $d_{5}$ given by (52).

Particular case 1: Neglecting the stretch effect $\left(\alpha_{0}=\lambda_{0}=\lambda_{1}=0\right)$, we obtain the expressions for displacement components, stresses and temperature field in a micropolar thermoelastic medium as given by $(53)-(58)$, only with $\Delta_{i}^{\prime}(i=1,2,4,5)$ replaced by $\Delta_{i}^{0}$, where

$$
\begin{array}{ll}
\Delta_{1}^{0}=-P_{0}\left[\xi_{5} a_{5}^{*} S_{2}-\xi_{4} a_{4}^{*} S_{1}\right], & \Delta_{2}^{0}=-P_{0}\left[\xi_{4} a_{4}^{*} S_{3}-\xi_{5} a_{5}^{*} S_{4}\right], \\
\Delta_{4}^{0}=-P_{0} \xi_{5} a_{5}^{*} b_{2}\left[f_{1} \xi_{2}-f_{2} \xi_{1}\right], & \Delta_{5}^{0}=P_{0} \xi_{4} a_{4}^{*} b_{2}\left[f_{1} \xi_{2}-f_{2} \xi_{1}\right] .
\end{array}
$$

For L-S and G-L theories, $d_{i}(i=1,2,5)$ are given by (51) and (52) respectively.

Particular case 2: Neglecting the microstretch effect, we obtain the expressions for displacement components, stresses and temperature field in a generalized thermoelastic medium as given by (60)-(64) for L-S and G-L theories, with $\Delta_{i}$ replaced by $\Delta_{i}^{\prime}(i=6,7,8)$, where

$$
\Delta_{6}^{\prime}=P_{0}\left(G_{2} H_{3}-H_{2} G_{3}\right), \quad \Delta_{7}^{\prime}=P_{0}\left(H_{1} G_{3}-G_{1} H_{3}\right), \quad \Delta_{8}^{\prime}=P_{0}\left(G_{1} H_{2}-H_{1} G_{2}\right) .
$$

\section{Numerical inversion of the transforms}

The solution of the problem is obtained by inverting the transforms in (43)-(49) for all the theories in case of mechanical and thermal sources. These expressions can be formally expressed as functions of $z$, the parameters of the Laplace and Hankel transforms $p$ and $q$ respectively and hence are of the form $\hat{f}(q, z, p)$. First we invert the Hankel transform, which gives the Laplace transform expression $\bar{f}(r, z, p)$ of the function $f(r, z, t)$ as

$$
\bar{f}(r, z, p)=\int_{0}^{\infty} q \hat{f}(q, z, p) J_{n}(q r) \mathrm{d} q .
$$

Now, for fixed values of $q, r$ and $z$, the function $\bar{f}(r, z, p)$ in (69) can be considered as the Laplace transform $\bar{g}(p)$ of the same function $g(t)$. Following Honig \& Hirdes (1984), the Laplace-transformed function $\bar{g}(p)$ can be inverted as given below:

$$
g(t)=\frac{1}{2 \pi i} \int_{C-i \infty}^{C+i \infty} e^{p t} \bar{g}(p) \mathrm{d} p,
$$

where $C$ is an arbitrary real number greater than all the real parts of the singularities of $\bar{g}(p)$. Taking $p=C+i y$, we get

$$
g(t)=\frac{e^{C t}}{2 \pi} \int_{-\infty}^{\infty} e^{i t y} \bar{g}(C+i y) \mathrm{d} y .
$$


Now, taking $e^{-C t} g(t)$ as $h(t)$ and expanding it as Fourier series in $[0,2 L]$, we obtain approximately the formula (Honig \& Hirdes 1984)

$$
g(t)=g_{\infty}(t)+E_{D}
$$

where

$$
g_{\infty}(t)=\left(C_{0} / 2\right)+\sum_{k=1}^{\infty} C_{k}, \quad 0 \leq t \leq 2 L
$$

and

$$
C_{k}=\left(e^{C t} / L\right) \operatorname{Re}\left[e^{i k \pi t / L} \bar{g}(C+(i k \pi / L))\right] .
$$

$E_{D}$ is the discretization error that can be made arbitrarily small by choosing a large enough $C$. The values of $C$ and $L$ are chosen according to the criteria outlined by Honig \& Hirdes (1984).

Since the infinite series in (73) can be summed up only to a finite number of $N$ terms, the approximate value of $g(t)$ becomes

$$
g_{N}(t)=\left(C_{0} / 2\right)+\sum_{k=1}^{N} C_{k}, \text { for } 0 \leq t \leq 2 L .
$$

Now we introduce a truncation error $E_{T}$ that must be added to the discretization error to produce the total approximate error in evaluating $g(t)$ using the above formula. Two methods are used to reduce the total error. The discretization errors is reduced by using the 'Korrecktur' method (Honig \& Hirdes 1984) and then the ' $\in$-algorithm' is used to reduce the truncation error and hence to accelerate the convergence. The Korrecktur method formula, to evaluate the function $g(t)$ is

$$
g(t)=g_{\infty}(t)-e^{-2 C L} g_{\infty}(2 L+t)+E_{D}^{\prime},
$$

where $\left|E_{D}^{\prime}\right|<<\left|E_{D}\right|$ (Honig \& Hirdes 1984).

Thus the approximate value of $g(t)$ becomes

$$
g_{N_{k}}(t)=g_{N}(t)-e^{-2 C L} g_{N^{\prime}}(2 L+t),
$$

where $N^{\prime}$ is an integer such than $N^{\prime}<N$.

We shall now describe the $\in$-algorithm, which is used to accelerate the convergence of the series in (75). Let $N$ be an odd natural number and $s_{m}=\sum_{k=1}^{m} C_{k}$ be the sequence of partial sums of (75). We define the $\in$-sequence by

$$
\epsilon_{0, m}=0, \quad \epsilon_{1, m}=s_{m}, \quad \epsilon_{n+1, m} \in_{n-1, m+1}+\frac{1}{\epsilon_{n, m+1}-\epsilon_{n, m}} ; \mathrm{n}, \mathrm{m}=1,2,3 \ldots
$$

It can be shown that (Honig \& Hirdes 1984), the sequence $\epsilon_{1,1}, \in_{3,1}, \ldots \ldots \ldots, \in_{N, 1}$ converges to $g(t)+E_{D}-\left(C_{0} / 2\right)$ faster than the sequence of partial sums $s_{m}, m=1,2,3 \ldots$ The actual procedure to invert the Laplace transform consists of (76) together with the $\in$ algorithm.

The last step is to evaluate the integral in (69). The method for evaluating this integral is described by Press et al (1986), which involves the use of Romberg's integration with adaptive step size. This also uses the results from successive refinements of the extended trapezoidal rule followed by extrapolation of the results to the limit when the step size tends to zero. 


\section{Discussion of numerical results}

We take the case of magnesium crystal (Eringen 1984) like material subject to mechanical and thermal disturbance for numerical calculations. The physical constants used by us are

$$
\begin{array}{lll}
\rho=1.74 \mathrm{gm} / \mathrm{cm}^{3}, & j=0.2 \times 10^{-15} \mathrm{~cm}^{2}, & \lambda=9.4 \times 10^{11} \mathrm{dyne} / \mathrm{cm}^{2} \\
\mu=4.0 \times 10^{11} \mathrm{dyne} / \mathrm{cm}^{2}, & K=1.0 \times 10^{11} \mathrm{dyne} / \mathrm{cm}^{2}, & \gamma=0.779 \times 10^{-4} \mathrm{dyne}, \\
\lambda_{0}=0.5 \times 10^{11} \mathrm{dyne} / \mathrm{cm}^{2}, & \lambda_{1}=0.5 \times 10^{11} \mathrm{dyne} / \mathrm{cm}^{2}, & \alpha_{0}=0.779 \times 10^{-4} \mathrm{dyne}, \\
K^{*}=0.6 \times 10^{-2} \mathrm{cal} / \mathrm{cm} \mathrm{s}^{\circ} \mathrm{C}, & C^{*}=0.23 \mathrm{cal} / \mathrm{gm}^{\circ} \mathrm{C}, & T_{0}=23^{\circ} \mathrm{C}, \\
t_{0}=6.131 \times 10^{-13} \mathrm{~s}, & t_{1}=8.765 \times 10^{-13} \mathrm{~s}, & \in=0.073, \epsilon_{1}=0.069 .
\end{array}
$$

Distributions of displacement, normal stress, tangential couple stress, microstress and temperature fields with radial distance $r$ for $\mathrm{L}-\mathrm{S}$ and G-L theories have been shown by (a) solid $(-)$ and dashed lines (--) respectively in generalized thermo-microstretch elastic (GTMSE) medium; (b) solid line with centred symbols (**) and dashed line with centred symbols $(-*-*-)$ respectively for generalized thermo-micropolar elastic (GTME) medium; (c) solid line with centred symbols $(-\odot)$ and dashed line with centred symbols $(-\circ-\circ-)$ respectively for generalized thermoelastic (GTE) medium. These distributions are shown graphically in figures $1-10$ for mechanical and thermal sources for $t=1.5 \mathrm{~s}$ and $z=1$. Some comparisons of both thermal theories and of the thermal (GTE), micropolar (GTME) and microstretch (GTMSE) theories are made.

\subsection{Case I (mechanical source)}

Behaviour of displacement for both the theories (L-S and G-L) in all the three media (GTMSE, GTME and GTE) is similar, whereas due to the stretch effect, the values of normal displacement in the GTMSE medium are slightly different as compared to those in the GTME medium for $\mathrm{L}-\mathrm{S}$ and $\mathrm{G}-\mathrm{L}$ theories, as shown in figure 1. Values of normal stress in the GTMSE

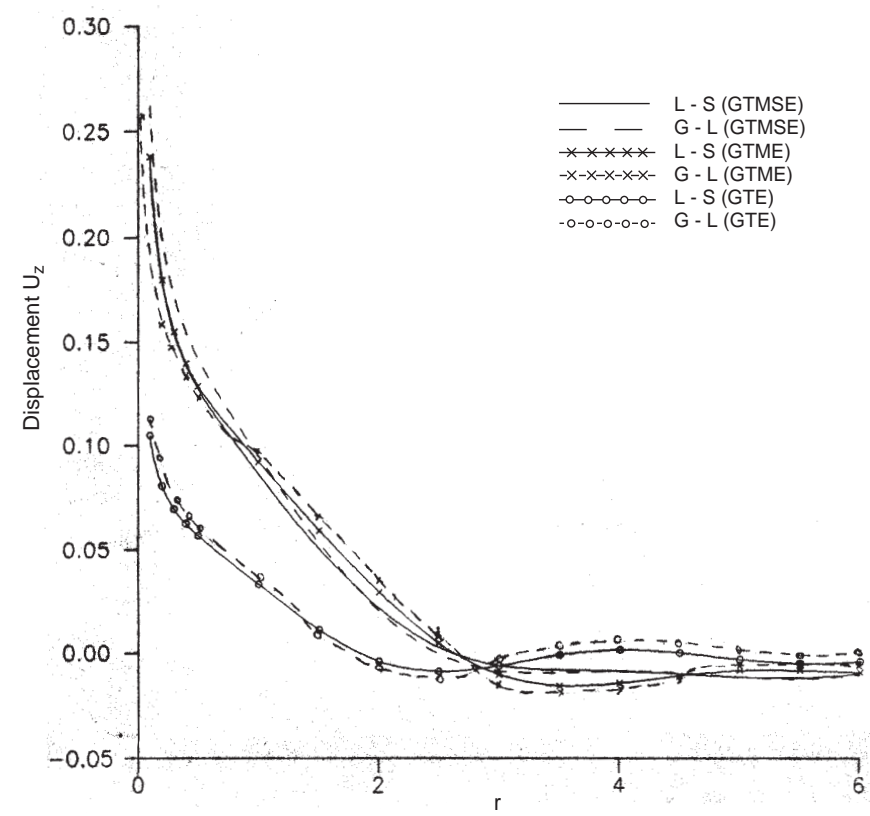

Figure 1. Variations of displacement $U_{\mathrm{z}}\left(=u_{\mathrm{z}} / P\right)$ with radial distance $r$ (mechanical source). 


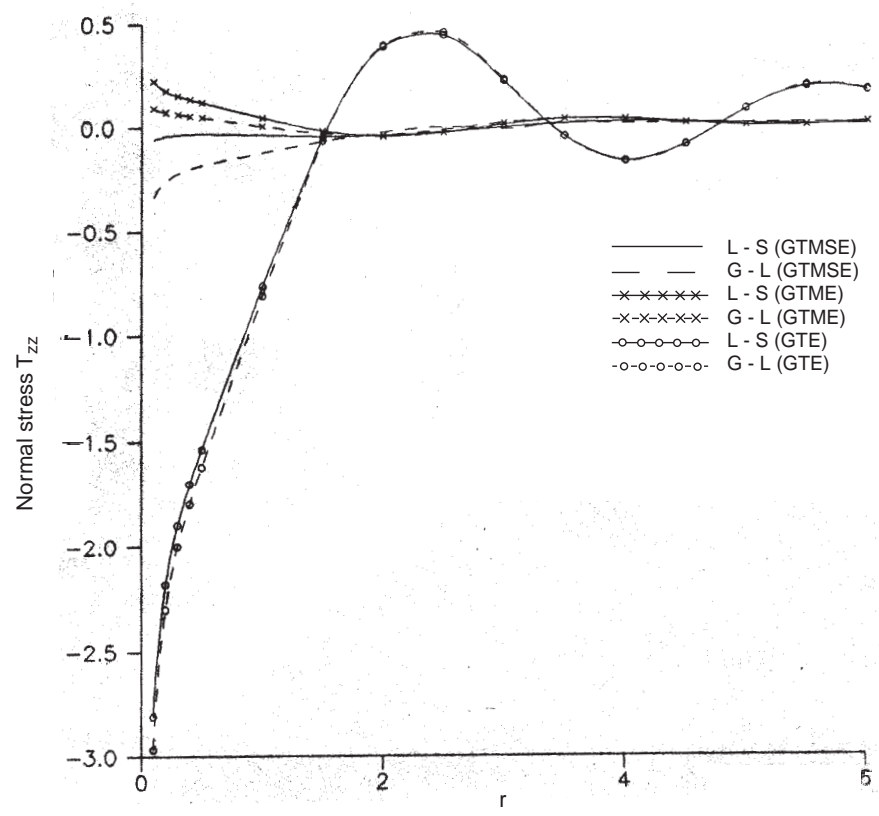

Figure 2. Variations of normal stress $T_{\mathrm{zz}}\left(=t_{\mathrm{zz}} / P\right)$ with radial distance $r$ (mechanical source).

medium are small compared to those in the GTME medium in the ranges $0<r \leq 1.8$ and $2.8 \leq r \leq 4.6$ but are large in the ranges $1.8<r<2.8$ and $4.6<r \leq 6.0$. Values of normal stress in the GTE medium are very small compared to those in the GTMSE and GTME media in the ranges $0<r<1.5$ and $3.6<r<4.8$, whereas the reverse happens in the other ranges. This distribution of normal stress for both the theories in all the three media has been shown in figure 2.

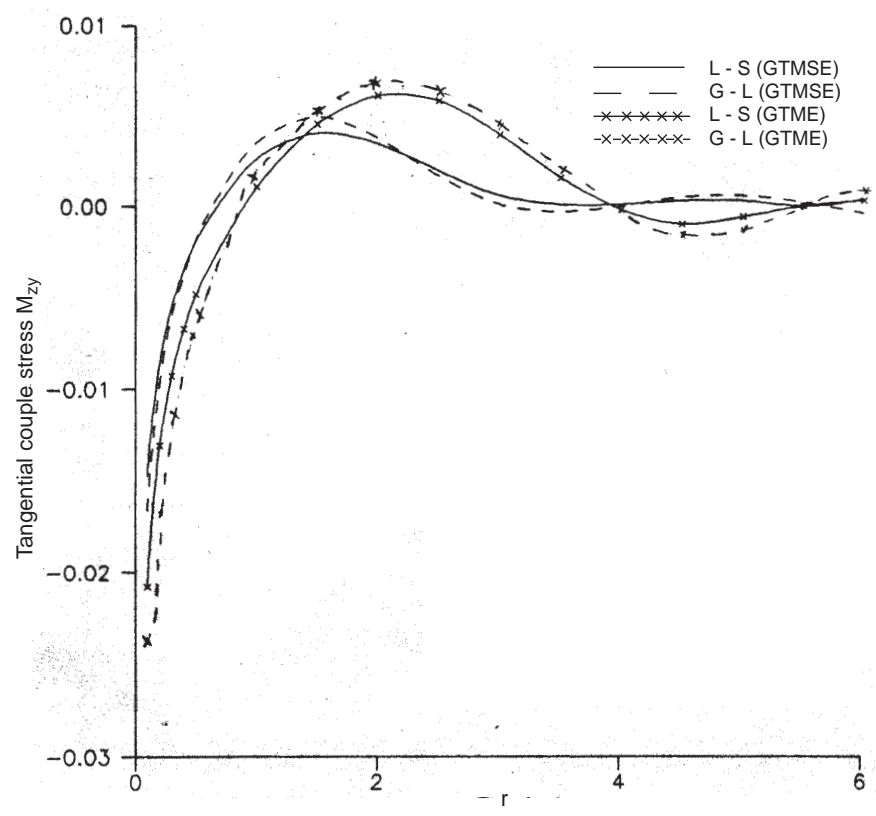

Figure 3. Variations of tangential couple stress $M_{\mathrm{zy}}\left(=m_{\mathrm{zy}} / P\right)$ with radial distance $r$ (mechanical source). 


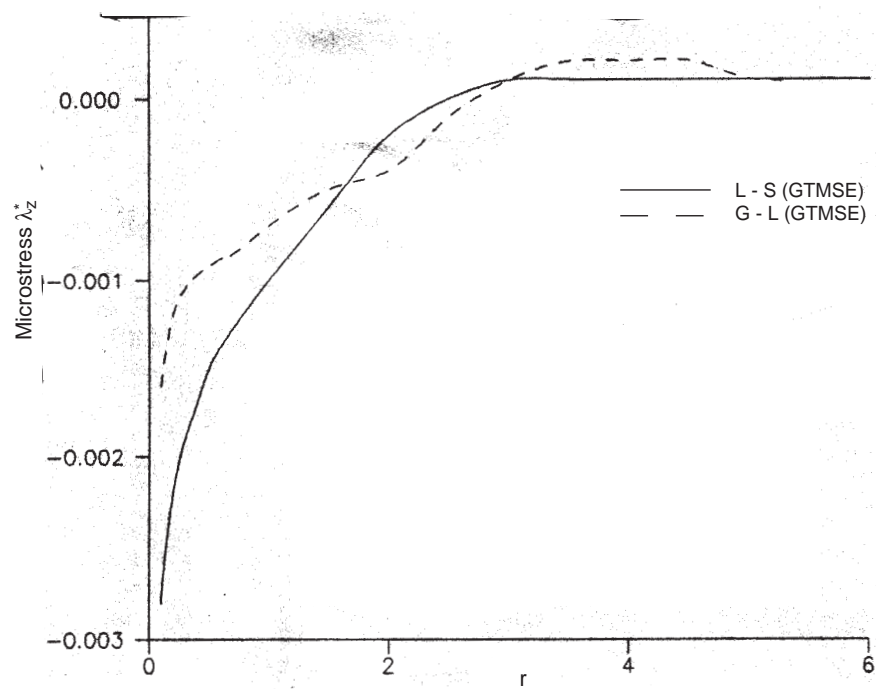

Figure 4. Variations of microstress $\lambda_{\mathrm{z}}^{*}\left(=\lambda_{\mathrm{z}} / P\right)$ with radial distance $r$ (mechanical source).

Stretch effect on tangential couple stress can be observed in figure 3 , where the values of tangential couple stress in the GTMSE medium are large in the ranges $0 \leq r \leq 1.5$ and $4.0 \leq r \leq 5.5$; small in the ranges $1.5<r<4.0$ and $5.5<r \leq 6.0$ as compared to those in the GTME medium for both the theories. Behaviour of microstress in the GTMSE medium is similar for both the theories, as shown in figure 4, where the values for G-L theory are large in comparison with those of the $\mathrm{L}-\mathrm{S}$ theory in the ranges $0 \leq r \leq 1.75$ and $3.0 \leq r \leq 5.0$, but are small in the range $1.75<r<3.0$, while values are the same for both the theories at $r>5.0$. Values of the temperature field are large in the GTMSE medium compared to those in the GTME and GTE media for both the theories as depicted in figure 5, where the values

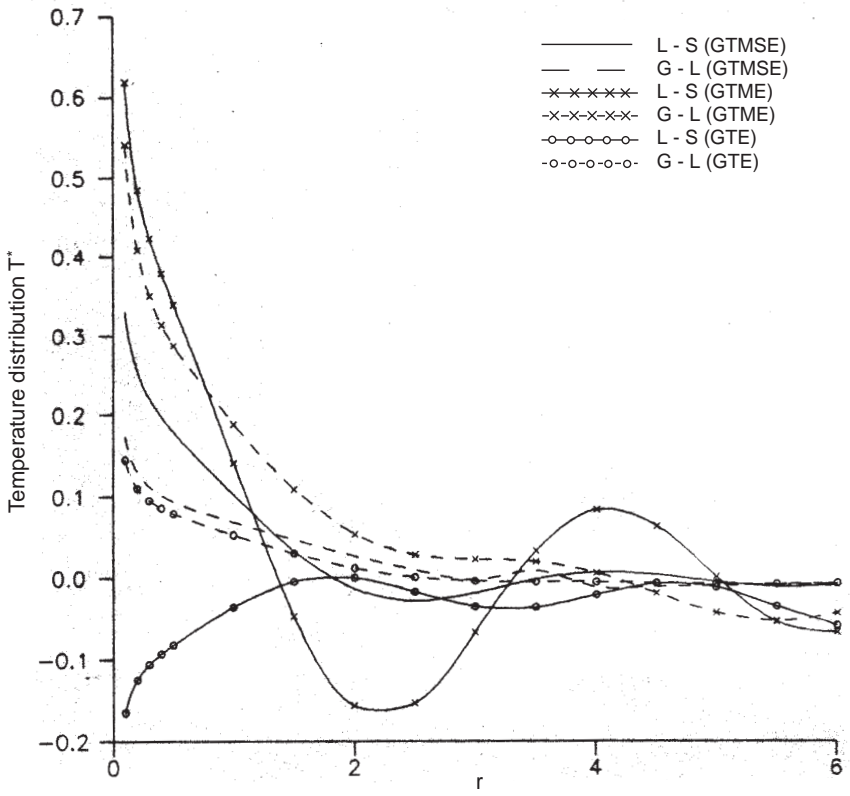

Figure 5. Variations of temperature distribution $T^{*}(=T / P)$ with radial distance $r$ (mechanical source). 


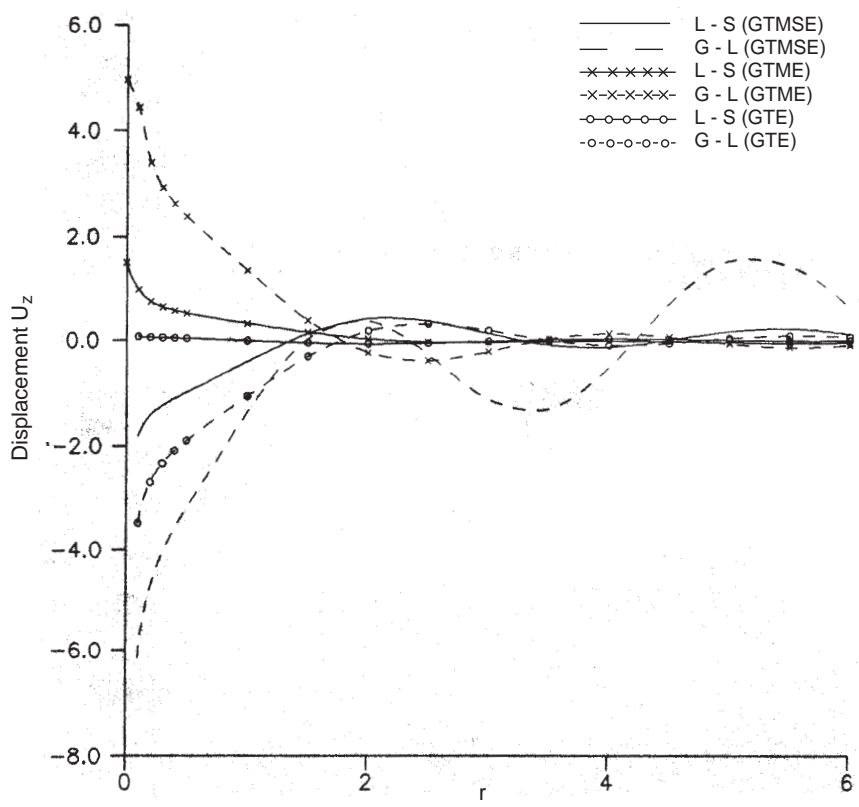

Figure 6. Variations of displacement $U_{\mathrm{z}}\left(=u_{\mathrm{z}} / P\right)$ with radial distance $r$ (thermal source).

of the temperature field in the GTME medium are multiplied by $10^{2}$ and $10^{3}$ for L-S and G-L theories respectively and in the GTE medium by $10^{2}$ for both the theories, to show the behaviour simultaneously.

\subsection{Case 2 (thermal source)}

Values of displacement are small in the GTMSE medium as compared to those in the GTME medium for both the theories and these variations have been shown in figure 6 after multiplying

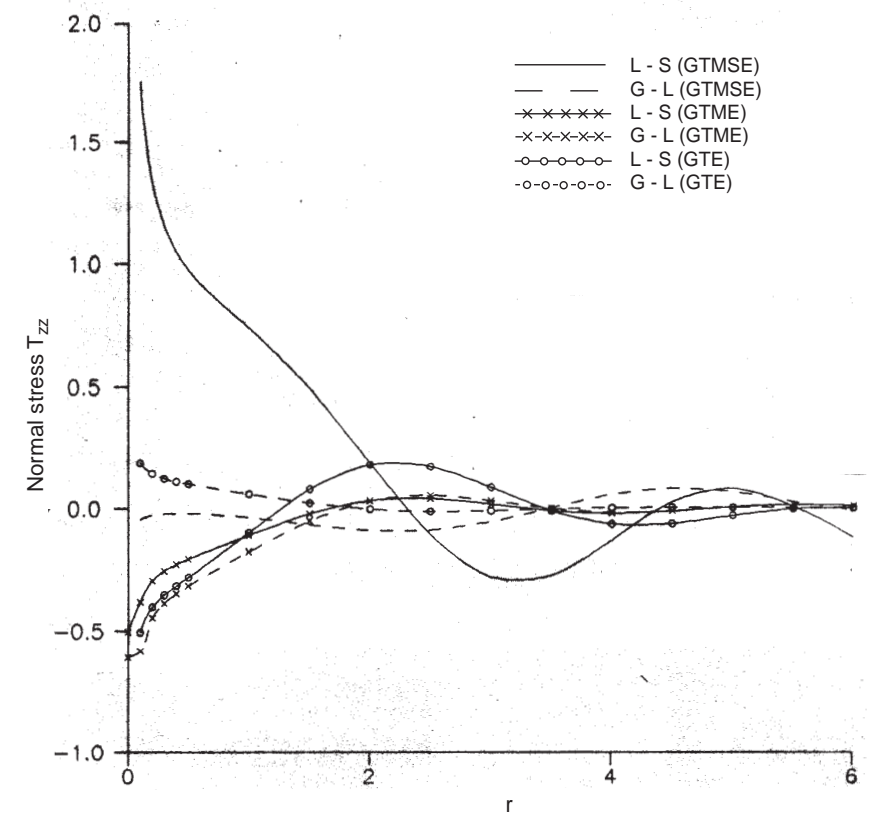

Figure 7. Variations of normal stress $T_{\mathrm{zz}}\left(=t_{\mathrm{zz}} / P\right)$ with radial distance $r$ (thermal source). 


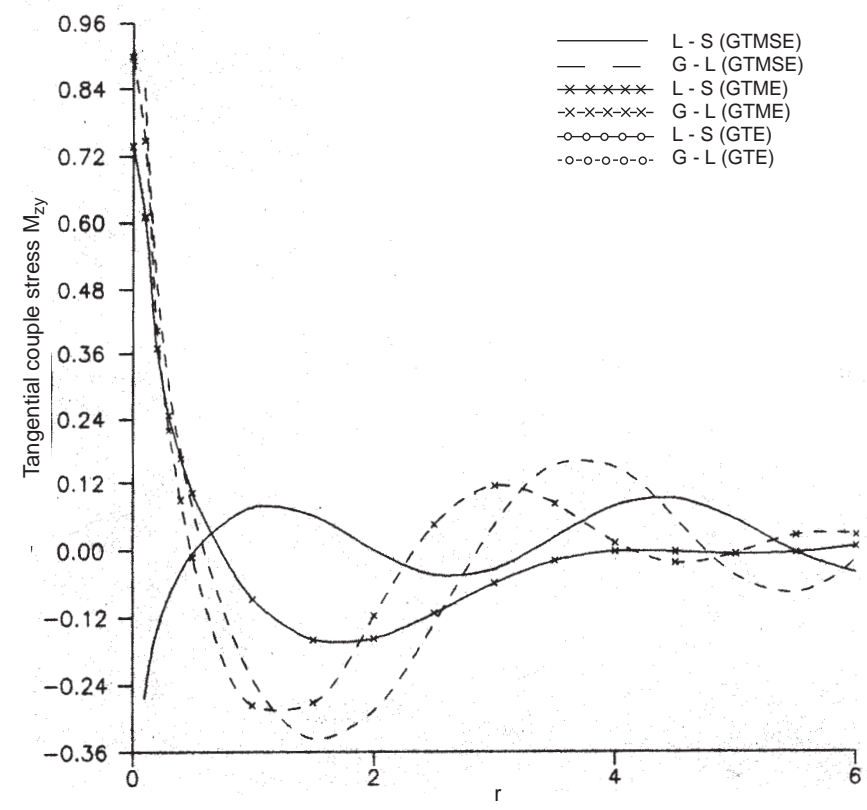

Figure 8. Variations of tangential couple stress $M_{\mathrm{zy}}\left(=m_{\mathrm{zy}} / P\right)$ with radial distance $r$ (thermal source).

the original values for the $\mathrm{L}-\mathrm{S}$ theory in both the media by 10 . It is also observed that the behaviour of displacement in the MSGTE medium is opposite to that in the MGTE medium for both the theories. Also the values of displacement for G-L theory in the GTE medium have been shown on dividing the original values by $10^{2}$. The distribution of normal stress for $\mathrm{L}-\mathrm{S}$ and $\mathrm{G}-\mathrm{L}$ theories is shown in figure 7 after dividing the original values for $\mathrm{G}-\mathrm{L}$ theory in MSGTE medium by $10^{2}$, in MGTE medium by 10 and in GTE medium by $10^{3}$, since the values for $\mathrm{G}-\mathrm{L}$ theory vary in a large range, compared to those of the $\mathrm{L}-\mathrm{S}$ theory.

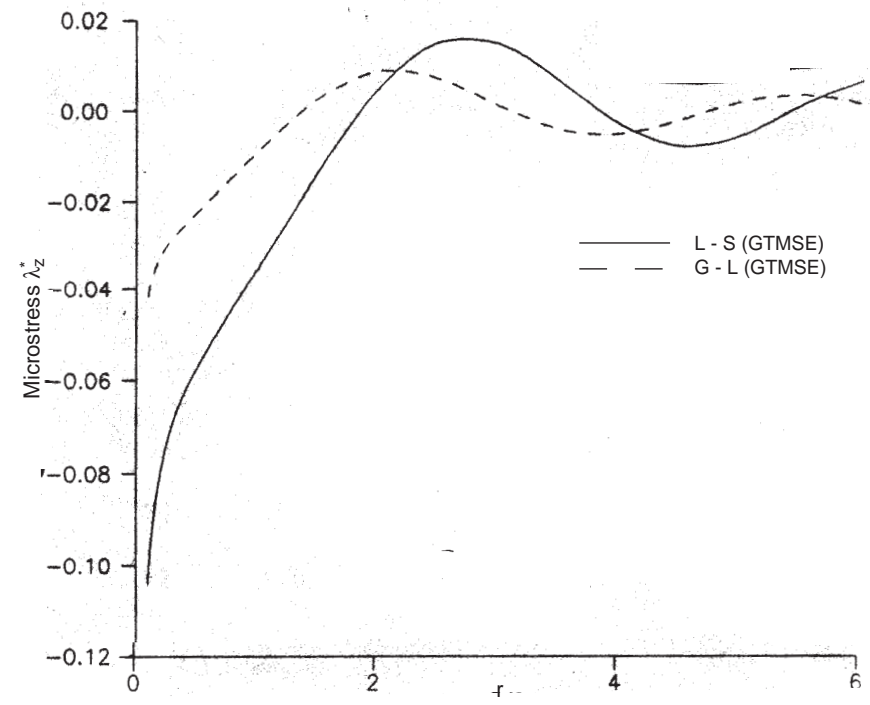

Figure 9. Variations of microstress $\lambda_{\mathrm{z}}\left(=\lambda_{\mathrm{z}} / P\right)$ with radial distance $r$ (thermal source). 


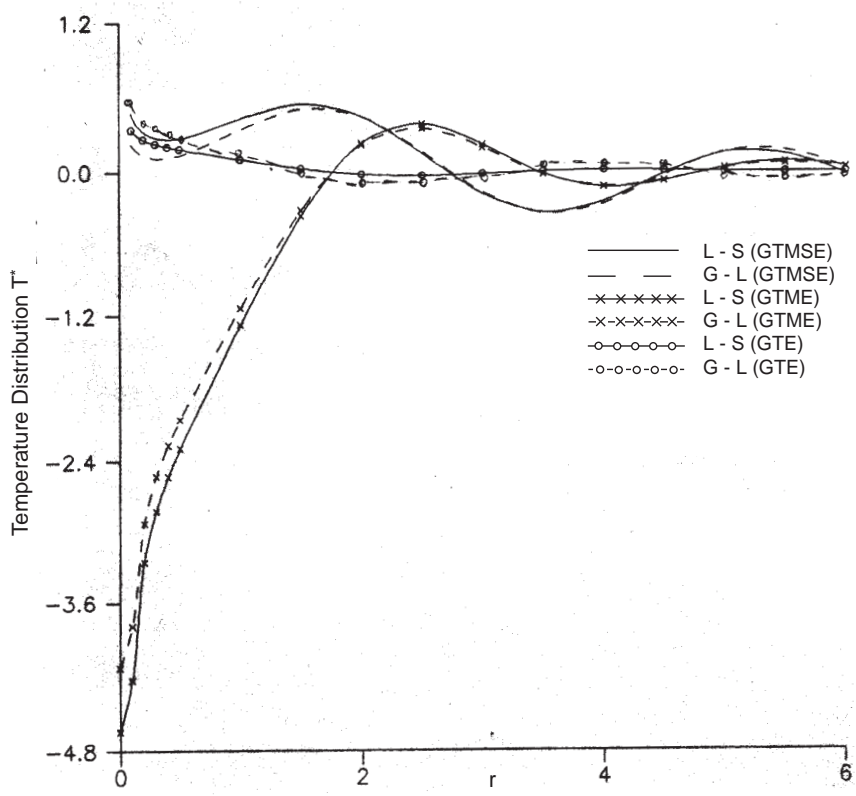

Figure 10. Variations of temperature distribution $T^{*}(=T / P)$ with radial distance $r$ (thermal source).

Distribution of tangential couple stress is illustrated in figure 8 after multiplying the original values for L-S theory in the GTMSE medium and the GTME medium by 10 and $10^{2}$ respectively and for G-L theory in the GTME medium by 10 to compare the behaviour of the results. Distribution of microstress in GTMSE medium for $\mathrm{L}-\mathrm{S}$ and G-L theories has been shown in figure 9 after dividing the original values for G-L theory by $10^{2}$. It is noticed that the range of distribution of microstress for $\mathrm{G}-\mathrm{L}$ theory is very large in comparison to that for $\mathrm{L}-\mathrm{S}$ theory, although the behaviour of microstress for the two theories is similar. The range of distribution of temperature field is small in GTMSE and GTE media for both the theories in comparison to GTME medium due to stretch and micropolar effects. Values of temperature field are large in the ranges $0 \leq r \leq 2.3$ and $4.5 \leq r \leq 6.0$ but small in the range $2.3<r<4.5$ in the GTMSE medium as compared to those in the GTME medium for both the theories. This distribution of temperature field is shown in figure 10.

\section{Conclusions}

It is evident from figures 1-10 that microstretch effects on displacement, normal stress, tangential couple stress, microstress and temperature field depend upon the radial distance, $r$. Also, for an instantaneous source (mechanical or thermal), the effects become smaller with increasing radial distance. Using these results, it is possible to investigate the disturbance caused by more general sources for practical applications.

\section{List of symbols}

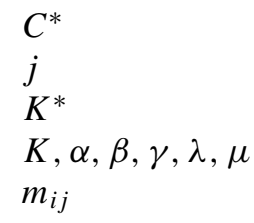

specific heat at constant strain; microrotational inertia; coefficient of thermal conductivity; micropolar material constants; components of couple stress tensor; 
$t_{i j} \quad$ components of force stress tensor;

$t_{0}, t_{1} \quad$ relaxation times;

$T \quad$ temperature variable;

$T_{0} \quad$ initial uniform temperature;

$\mathbf{u}, \boldsymbol{d} \quad$ displacement and microrotation vector respectively;

$\alpha_{t_{1}}, \alpha_{t_{2}} \quad$ coefficients of linear expansion;

$\alpha_{0}, \lambda_{0}, \lambda_{1} \quad$ stretch constants;

$\delta_{1 k} \quad$ Kronecker delta;

$\nabla \quad$ del operator;

$\in \quad$ thermo coupling coefficient;

$\epsilon_{i j r} \quad$ alternate tensor;

$\lambda_{k} \quad$ microstress;

$\nu, \nu_{1} \quad$ thermal constants;

$\rho$ density of generalized thermo-microstretch elastic solid;

$\phi^{*} \quad$ scalar microstretch function

\section{References}

Bofill F, Quintanilla R 1995 Some qualitative results for the linear theory of thermo-microstretch elastic solids. Int. J. Eng. Sci. 33: 2115-2125

Chandrasekharaiah D S 1999 Thermoelasticity with thermal relaxation: An alternative formulation. Proc. Indian Acad. Sci. (Math. Sci.) 109: 95-106

Chandrasekharaiah D S, Srinath K S 2000 Thermo elastic waves without energy dissipation in an unbounded body with a spherical cavity. Int. J. Math. Math. Sci. 23: 555-562

Ciarletta M 1999 On the bending of microstretch elastic plates. Int. J. Eng. Sci. 37: 1309-1318

De Cicco S, Nappa L 1997 Torsion and flexure of microstretch elastic circular cylinders. Int. J. Eng. Sci. 35: 573-587

De Cicco S, Nappa L 1999 On the theory of thermomicrostretch elastic solids. J. Thermal Stresses 22: $565-580$

Eringen A C 1966a Linear theory of micropolar elasticity. J. Math. Mech. 15: 909-923

Eringen A C 1966b Theory of micropolar fluids. J. Math. Mech. 16: 1-18

Eringen A C 1970 Foundation of micropolar thermoelasticity; Course of lectures no. 23, CISM(Udine: Springer)

Eringen A C 1971 Micropolar elastic solids with stretch. Ari Kitabevi Matbassi 24: 1-18

Eringen A C 1976 Nonlocal polar field theories. In Continuum physics (ed.) A C Eringen (New York: Academic Press) vol. 4, pp. 205-267

Eringen A C 1984 Plane waves in non-local micropolar elasticity. Int. J. Eng. Sci. 22: 1113-1121

Eringen A C 1990a Theory of thermo-microstretch fluids and bubbly liquids. Int. J. Eng. Sci. 28: $133-143$

Eringen A C 1990b Theory of thermo-microstretch elastic solids. Int. J. Eng. Sci. 28: 1291-1301

Green A E, Lindsay K A 1972 Thermoelasticity. J. Elasticity 2: 1-5

Honig G, Hirdes U 1984 A method for the numerical inversion of the Laplace transform. J. Comput. Appl. Math. 10: 113-132

Iesan D 1997 Uniqueness results in the theory of microstretch fluids. Int. J. Eng. Sci. 35: 669-679

Iesan D, Nappa L 1995 Extension and bending of microstretch elastic circular cylinders. Int. J. Eng. Sci. 33: 1139-1151

Iesan D, Pompei A 1995 On the equilibrium theory of microstretch elastic solids. Int. J. Eng. Sci. 33: 399-410

Iesan D, Quintanilla R 1994 Existence and continuous dependence results in the theory of microstretch elastic bodies. Int. J. Eng. Sci. 32: 991-1001 
Lord H W, Shulman Y 1967 A generalized dynamical theory of thermoelasticity. J. Mech. Phys. Solid 15: 299-306

Nowacki W 1966 Couple stresses in the theory of thermoelasticity. Proc. IUTAM Symposia (Vienna: Springer-Verlag) pp 259-278

Press W H, Teukolsky S A, Vellerling W T, Flannery B P 1986 Numerical recipes (Cambridge: University Press)

Singh B, Kumar R 1998 Wave propagation in a generalized thermo-microstretch elastic solid. Int. J. Eng. Sci. 36: 891-912 